\title{
Evaluación del vocabulario controlado DeCS en logopedia desde la perspectiva del usuario: estudio de observación de la recuperación de información con protocolo verbal
}

\author{
Vera Regina Casari Boccato \\ Universidad de São Paulo (Brasil) \\ Mariângela Spotti Lopes Fujita \\ Universidad Estadual Paulista de Marília (Brasil)
}

\section{Resumen}

Se evalúa, por medio de la observación del usuario, el lenguaje de indización DeCS (Descriptores en Ciencias de la Salud), utilizado para la recuperación de información en el sistema LILACS (Literatura Latinoamericana y del Caribe sobre Ciencias de la Salud), con la finalidad de obtener indicadores para diseñar las estrategias de perfeccionamiento del lenguaje en el área de la logopedia. Para la evaluación de este lenguaje se empleó la técnica del protocolo verbal o "pensar en voz alta" (thinking aloud), teniendo como sujetos investigadores del Departamento de Logopedia de la Facultad de Odontología de Bauru, de la Universidad de São Paulo-FOB-USP (Brasil). La aplicación de esta técnica fue realizada en el propio ambiente de trabajo de los sujetos, habiendo verificado anteriormente el conocimiento de cada uno de ellos en cuanto al acceso al sistema LILACS, así como a la utilización del lenguaje DeCS. Se seleccionó y observó con el protocolo verbal a cuatro personas, representantes de las cuatro especialidades formadoras del área de la logopedia: lenguaje, voz, motricidad oral y audiología, en la tarea de llevar a cabo la recuperación de información en el campo de descriptor de asunto de la interfaz de búsqueda de la base de datos LILACS, que utiliza el lenguaje DeCS. El análisis de las transcripciones de los protocolos verbales demostró que la metodología empleada fue eficaz por posibilitar la recogida, en tiempo real, de las declaraciones de los sujetos (usuarios/investigadores) sobre el funcionamiento del lenguaje DeCS, empleado para la recuperación de informaciones en el sistema LILACS. El estudio llevó a una reflexión sobre las declaraciones emitidas por los cuatro participantes en nuestra investigación y los resultados obtenidos del análisis revelan que el lenguaje DeCS, en logopedia, condujo las búsquedas a resultados insatisfactorios en cuanto a la recuperación de información a partir de los siguientes aspectos relevantes: insuficiencia de términos genéricos y/o específicos

Scire. $12: 1$ (en.-jun. 2006) 179-195. ISSN 1135-3761. 
representativos del área de la logopedia; necesidad de actualización de términos disponibles en el lenguaje, con relación a la terminología encontrada en la literatura científica del área y adoptada por los especialistas; y jerarquización de términos en categorías de asuntos no equivalentes a sus conceptos, entre otros. Se concluye que el perfeccionamiento del lenguaje documental DeCS en el área de la logopedia requiere la actualización de los descriptores y definiciones correspondientes, conforme a la realidad de avance científico del área; revisión de la traducción al portugués de los descriptores existentes en el vocabulario DeCS y establecimiento de las relaciones de equivalencia, jerarquía y no jerarquía para todos los descriptores. Por otro lado, la terminología del área de la logopedia utilizada por especialistas, entidades de investigación y literatura científica debe representar a las especialidades de lenguaje, voz, motricidad oral y audiología. Se recomienda al BIREME (Centro Latinoamericano y del Caribe de Información sobre Ciencias de Salud), institución responsable de la elaboración del vocabulario controlado DeCS, la construcción de una categoría específica para el área de la logopedia que represente a la literatura y la comunidad científica brasilera del área, un ejemplo de inclusión de las categorías de Salud Pública y Homeopatía, teniendo en cuenta que su representación terminológica no confiere las necesidades de los usuarios/investigadores para la recuperación de informaciones.

Palabras clave: Lenguaje de indización. Evaluación. Sistema de información. Recuperación de información. Protocolo verbal. Logopedia.

\section{Abstract}

Assessment of the DeCS (Health Sciences Descriptors) indexing language, utilized for the retrieval of information in the LILACS (Latin American and Caribbean Health Sciences) system, aiming at obtaining indicators to outline strategies to improve the language in the field of Speech-Language Pathology and Audiology. The verbal protocol technique (thinking aloud) was utilized to evaluate this language, having as subjects researchers of the Speech-Language Pathology and Audiology Department at the Dental School, University of São Paulo-FOB-USP, at Bauru, SP. This technique has been applied in the subjects' own working environment, being their knowledge on the access to LILACS system as well as the use of DeCS language previously verified. Then, four subjects representing the four forming specialties in the area of Speech-Language Pathology and Audiology, i. e., Language, Voice, Oral Motricity and Audiology, were selected and observed, with the verbal protocol, in the task of performing information retrieval, in the field of subject descriptor of the search interface of LILACS database, utilizing the DeCS language. The analysis of the transcriptions for the verbal protocols demonstrated that the methodology employed was effective in enabling the collection, in real time, of the subjects' statements (users /researchers) on the performance of DeCS

Scire. $12: 1$ (en.-jun. 2006) 179-195. ISSN 1135-3761. 
language utilized for information retrieval in the LILACS system. The study carried out a reflection on the declarations issued by the four subjects participating in our research and the results obtained from the analysis show that the DeCS language, in Speech-Language Pathology and Audiology, led the searches to unsatisfactory results as to information retrieval from the following relevant aspects: insufficiency of generic and/or specific terms representative of the SpeechLanguage Pathology and Audiology area; terms available in the language regarding the terminology found in the scientific literature of the area and adopted by specialists need to be updated; ranking of terms in subject categories not equivalent to their concepts, among others. It is concluded that the improvement of the DeCS indexing language in the field of Speech-Language Pathology and Audiology requires the updating of descriptors and corresponding definitions according to the reality of scientific advancement in the area, review of the translation for the Portuguese language, of descriptors existing in the DeCS Vocabulary, and the establishment of hierarchical and nonhierarchical equivalence relations to all descriptors. On the other hand, the survey of terminology in the area of Speech-Language Pathology and Audiology utilized by specialists, research societies and scientific literature must represent the specialties of Language, Voice, Oral Motricity and Audiology. It is recommended that the Latin-American and Caribbean Health Science Information Center-BIREME, an institution responsible for the compilation of DeCS Controled Vocabulary, elaborates a specific category for the area of Speech-Language Pathology and Audiology, representing the literature and the Brazilian scientific community in the area, such as the inclusion of Public Health and Homeopathy, since its terminological representation is not consistent with the needs of users/researchers engaged in information retrieval.

Keywords: Indexing language. Evaluation. Information system. Information retrieval. Verbal protocol. Speech-Language Pathology and Audiology.

\section{Introducción}

En los últimos veinte años, la comunidad científica viene atribuyendo a la investigación la responsabilidad de ser una importante herramienta para la generación de conocimiento que contribuye a la mejoría de la situación de la salud de la población brasilera, así como a las tomas de decisión con respecto a planificación, programas de salud y generación de conocimientos (Brasil, 2004).

En cuanto al área de la logopedia, ésta realiza investigaciones importantes que viabilizan el desarrollo científico, con estudios sobre los problemas de la comunidad humana, con la intención de nuevos descubrimientos y directrices en la adopción de técnicas de evaluación, de terapia, de actitudes preventivas dirigidas al tratamiento y/o rehabilitación de pacientes con problemas auditivos o de lenguaje, entre otras patologías. En este sentido, las universidades y sus respectivas unidades de

Scire. $12: 1$ (en.-jun. 2006) 179-195. ISSN 1135-3761. 
información tienen un papel fundamental en la generación, diseminación y difusión de ese conocimiento.

Un sistema de información debe reflejar las necesidades del usuario/investigador en la búsqueda de datos que afecten a sus investigaciones.

El lenguaje documental, como instrumento mediador entre la información documental, el sistema de información y el usuario, tiene la función de facilitar el acceso a esa información, la satisfacción en la recuperación de información y, consecuentemente, la creación del conocimiento científico, posibilitando el bienestar de la sociedad.

Entretanto, el lenguaje documental no se presenta en concordancia con las peticiones de búsqueda realizadas por el usuario; muchas veces la calidad de las investigaciones realizadas y la credibilidad del sistema de información son discutibles.

El propósito de este estudio es, por lo tanto, evaluar mediante la observación del usuario, en el área de la logopedia, el lenguaje documental DeCs (1) del sistema de información LILACS (2), producido por el BIREME (3), con la aplicación de la técnica del protocolo verbal o "pensar en voz alta" (thinking aloud) con el objetivo de contribuir a la recopilación de indicadores que conducirán las estrategias de perfeccionamiento del lenguaje en logopedia.

\section{El sistema de información LILACS y el lenguaje DeCS en la perspectiva de la comunicación científica}

Buscando la calidad y el perfeccionamiento de los servicios de atención a los usuarios de la comunidad científica del área de salud, el BIREME (Centro Latinoamericano y del Caribe de Información sobre Ciencias de la Salud) desarrolla un trabajo conjunto con más de 500 bibliotecas y centros colaboradores que integran el Sistema Latinoamericano y del Caribe de Información sobre Ciencias de la Salud, representado por 37 países, en la indización de documentos en fuentes de información como la base de datos LILACS.

Ese trabajo en colaboración proporciona la visibilidad de la producción científica de cada institución integrante del sistema, además de posibilitar el intercambio con servicios que amplíen ese acceso a la información en el área de la salud, beneficiando a los propios usuarios del sistema latinoamericano.

Uno de los objetivos del BIREME, principal sistema de información latinoamericano en el área de la salud, es

coordinar, operar y promover el control bibliográfico, la divulgación, la evaluación y el mejoramiento de la literatura científico-técnica publicada en papel y en formato electrónico en los países de la REGIÓN, la cual deberá ser indizada en las bases de datos del sistema LILACS (Literatura Latinoamericana y del Caribe de Información sobre Ciencias de la Salud), que incluyen la base de datos regional LILACS, las bases de datos 
nacionales que representan la memoria de la literatura científico-técnica de los países y las bases de datos especializadas, producidas y operadas por la OPAS y por los países. (BIREME, 2004a)

El BIREME, desde su fundación en 1967 hasta la actualidad, por medio de convenios realizados entre la OPAS (Organización Panamericana de la Salud), el Gobierno brasilero representado por los Ministerios de Salud y de Educación, la Secretaría de Salud del Estado de São Paulo y la Escuela Paulista de Medicina, viene realizando sus servicios teniendo en cuenta el desarrollo de la salud por medio del fortalecimiento y de la ampliación del flujo de informaciones en ciencias de la salud.

La necesidad de reordenación de los productos y servicios tradicionales para acompañar a las necesidades informacionales de los investigadores del área de salud ante los avances tecnológicos actuales orientó al BIREME, en 1998, a oficializar la creación e implantación de la BVS (Biblioteca Virtual de la Salud), disponible vía Internet en http://www.bireme.br y accesible a todos los usuarios de la comunidad científica nacional e internacional del área de ciencias de la salud.

Dentro de esos servicios on-line, el sistema de información/base de datos LILACS tiene como finalidad el control de la producción bibliográfica y difusión de la literatura científica latinoamericana y del Caribe en el área de ciencias de la salud (medicina, medicina veterinaria, odontología, salud pública, psicología y logopedia, entre otras), con la descripción formal y de contenido de tesis, libros, capítulos de libros, actas de congresos o conferencias, informes científico-técnicos, artículos de revistas, materiales no convencionales, etcétera, de autores latinoamericanos y caribeños, publicados en los países de la zona a partir de 1982.

Esa base de datos se originó a partir del IMLA (index medicus latinoamericano), iniciado en 1979. Posteriormente, EL BIREME desarrolló su sistema LILACS, basado en la experiencia adquirida por la base IMLA y compatible con las existentes y utilizadas por los centros colaboradores de la red para la entrada y recuperación de información.

El lenguaje de recuperación adoptado por el sistema LILACS es un vocabulario estructurado DeCS (Descriptores en Ciencias de la Salud). "Los vocabularios estructurados son colecciones de términos organizados según una metodología en la cual es posible especificar las relaciones entre conceptos con el propósito de facilitar el acceso a la información" (BIREME, 2004b).

El vocabulario estructurado DeCS, tiene la finalidad de constituir un lenguaje único para la indización y recuperación de documentos en la base de datos LILACS por parte del Sistema Latinoamericano y del Caribe de Información sobre Ciencias de la Salud.

Para su elaboración se tomó como base el lenguaje documental MeSH (Medical Subject Headings), producido por la NLM (National Library of Medicine), 
publicado en 1960 y actualizado en 1963, para la indización y recuperación de artículos de periódicos publicados en Estados Unidos y en más de 70 países, y disponibles en la base de datos MEDLINE (United States National Library of Medicine, 2005).

La primera edición de DeCS tuvo lugar en el año 1987, en formato impreso, y se presentó en dos volúmenes constituidos por las listas alfabética y jerárquica en los idiomas portugués y español. Después se publicaron otras dos ediciones.

A partir del año de 1999, el vocabulario DeCS, formado por las listas alfabética, permutada y jerárquica, estuvo disponible en su versión on-line (DeCS Home), vía Internet, en http://decs.bvs.br/.

Dentro de los lenguajes documentales alfabéticos, el DeCS, como el MeSH, es considerado un tesauro que, siguiendo la tradición de los sistemas de clasificación y de las listas de encabezamientos de materia, fue transformándose en un vocabulario controlado especializado, aunque deja de utilizar las estructuras de dichos sistemas de clasificación, de las cuales constituye el origen.

Su estructura jerárquica es fundamental en la división del conocimiento en clases y subclases decimales; respetando las relaciones conceptuales y la semántica, sus términos son presentados en una estructura híbrida de pre y poscoordinación para la indización y recuperación de información.

De esta forma, DeCS es un vocabulario traducido del MeSH para los idiomas portugués y español que presenta los términos que lo componen en tres idiomas (inglés, portugués y español). La estructura y la organización de los descriptores fueron mantenidas tal como aparecían en el MeSH.

Para atender a las necesidades de indización y recuperación de información de los centros colaboradores de algunas áreas no representativas y/o inexploradas por la literatura y, consecuentemente, por las bases de datos internacionales, fue necesaria la creación de dos categorías representativas de áreas temáticas de salud pública y homeopatía, no existentes en el lenguaje MeSH.

DeCS es un lenguaje multijerárquico que posibilita a un mismo descriptor estar incluido en más de una categoría. Se dan relaciones lógico-semánticas entre los términos, pero no siempre de acuerdo con las directrices internacionales establecidas (Austin y Dale, 1993).

En relación con el área objeto de este estudio, la logopedia, está formada por las especialidades de lenguaje, voz, motricidad oral y audiología (Conselho Federal de Fonoaudiologia, 2001). El vocabulario DeCS no posee una categoría específica para logopedia, de modo que esta se encuentra incluida dentro de la categoría de salud pública. Por el hecho de ser un área multidisciplinar, sus términos también están clasificados en varias otras categorías del vocabulario, siendo las principales anatomía, enfermedades, técnicas y equipamientos, psicología y psiquiatría, antropología, educación, sociología y fenómenos sociales, entre otras. 
El término logopedia no existe en Estados Unidos ni en Europa debido a que la estructura del área es diferente de la de los países de Latinoamérica. El término speech-language pathology engloba los estudios de las especialidades del habla (speech), el lenguaje (language), voz (voice) y motricidad oral (expresión —oral motricity - también inexistente en Estados Unidos). La audiología es una especialidad independiente y representada por el término audiology.

En este sentido, el término logopedia, que no existe en el vocabulario de la lengua inglesa, fue traducido en DeCS como speech, language and hearing sciences y, de acuerdo con la literatura de la logopedia, todavía no está claro que esa sea la traducción más adecuada.

\section{Evaluación del lenguaje documental: presupuestos teóricos}

La actuación eficaz de un lenguaje documental en la recuperación de información es un factor esencial en la obtención de datos satisfactorios para el usuario/investigador, ya que posibilita la realización de investigaciones que contribuyan al desarrollo de la logopedia y de la ciencia brasilera, intentando mejorar la calidad de vida de la comunidad.

La búsqueda de la información deseada por el usuario y la obtención de esta de acuerdo con el asunto de la investigación implica un trabajo conjunto entre el sistema, un lenguaje documental y el propio usuario.

Dentro de este contexto, se han llevado a cabo muchos estudios para la evaluación de lenguajes documentales por el uso, por la forma y/o por el contenido, aplicando enfoques cuantitativos, cualitativos y/o cognoscitivos, con la intención de verificar su papel en los sistemas de información para la recuperación de la misma.

En este campo de estudio destacan investigadores como Cleverdon (1964) y Lancaster (2004) en el desarrollo de trabajos que definen los criterios y los índices cuantitativos de precisión (precision ratio) y acierto (recall ratio), que contribuyeron a la evaluación de los lenguajes con el establecimiento de parámetros metodológicos.

Dentro de ese enfoque cuantitativo, también merecen especial atención otros trabajos internacionales sobre la evaluación del lenguaje según el uso, como las pruebas de ASTIA (Armed Services Technical Information Agency), los proyectos Cranfield I y II, el programa de evaluación desarrollado por F. W. Lancaster para una evaluación del funcionamiento del sistema Medlars, la prueba de Aberystwyth, que abarca estudios sobre lenguajes naturales y controlados, y los estudios de indización sistemática de J. Kaiser, complementados posteriormente por las pruebas realizadas por la WRU (Western Reserve University), que se centran en la evaluación formal del lenguaje (Foskett, 1973; Melo, 1994).

Scire. $12: 1$ (en.-jun. 2006) 179-195. ISSN 1135-3761. 
Las investigaciones en Brasil también ofrecieron resultados muy significativos sobre la evaluación formal del lenguaje, como el realizado por Lara (1993) en su tesis doctoral y el de Strehi (1998), con el análisis del vocabulario controlado que se utiliza en la base de datos de una biblioteca universitaria de arte.

En el área de la salud, el vocabulario estructurado DeCS también fue objeto de los estudios de algunos investigadores internacionales y nacionales, en la verificación de su performance, de su estructura de indización y recuperación de información en sistemas de información. Así se presentan los trabajos desarrollados por los estudiosos cubanos Valdés Abreu (1996), Rodríguez Camiño (1998), Jiménez Miranda (1998, 2002) y Pellizzon (2004), así como los realizados por los investigadores argentinos Otero et al. (2004).

El trabajo brasilero realizado por Santos (2002) también contribuyó al perfeccionamiento del lenguaje DeCS, con el desarrollo de un estudio de evaluación en la categoría de salud pública, utilizado para la indización y recuperación de información en la base de datos AdSaúde (Administración de Salud), del sistema especializado en administración de salud del BIREME.

En el ámbito de las investigaciones de evaluación centradas en el usuario, los estudios cualitativos basados en percepciones y/o en opiniones deben ser considerados fundamentales, ya que las actitudes y las opiniones emitidas por el usuario deben ser consideradas de suma importancia para el perfeccionamiento de los servicios de recuperación de información y, consecuentemente, de los lenguajes documentales utilizados por el sistema.

Esas investigaciones, que utilizan enfoques cognitivos, fueron desarrolladas en diversas áreas del conocimiento, principalmente en la de las ciencias de la información, a partir de la década de 1970.

Los trabajos pioneros de Ericsson y Simon (1987), con la utilización de la técnica del protocolo verbal en la observación de la actividad de lectura, también colaboraron con sus resultados a esos estudios de observación.

Para la verificación de la eficacia de la recuperación de información y la interacción del usuario, en especial los aspectos cognitivos del proceso, Ingwersen (1982) realizó una evaluación cualitativa utilizando el protocolo verbal en el servicio de referencia de una biblioteca pública. Según este investigador, los sujetos eran trece bibliotecarios: siete de ellos ocupaban cargos de dirección, tres de referencia y tres trabajaban en ambas actividades. Los trece tenían varios años de experiencia en la biblioteca, y los centros escogidos cubrían tanto grandes ciudades como áreas rurales. Los cinco usuarios que participaron fueron escogidos entre las personas con una experiencia limitada en el uso de la biblioteca: cuatro técnicos y un estudiante universitario. 
Se prestó mucha atención a la instrucción de los sujetos antes de las pruebas, lo que supone la familiarización de los trece sujetos con la técnica y entrenamientos sobre cómo "pensar en voz alta", es decir, cómo exteriorizar sus pensamientos en el momento de la ejecución de la actividad de recuperación de información y durante la grabación, y también sobre la utilización del sistema de información. Complementando la aplicación de la técnica, el autor también observó el comportamiento de los sujetos durante todo el proceso. Realizó un estudio sobre la negociación que tuvo lugar durante la investigación del sistema de información entre el usuario y el bibliotecario.

Se realizaron siete grabaciones que cubrían la búsqueda del usuario, la negociación y la búsqueda de información. Cada grabación se basó en las necesidades del usuario participante acerca de las siguientes cuestiones: 1) las identidades del álgebra booleana en la forma adecuada para la aplicación, creación y reparación de los circuitos en los computadores; 2) la literatura no tendenciosa sobre el pacto germano-soviético de 1939 en forma de fuentes de información, correspondencia, textos sobre el pacto, con especial consideración al esclarecimiento de en qué lado se originó la iniciativa.

Cada registro se reprodujo en un protocolo verbal, para lo cual se incluyeron algunos hechos ocurridos, así como las observaciones referentes al comportamiento de los sujetos involucrados. Se añadieron comentarios adicionales de su propia observación.

La técnica introspectiva del protocolo verbal o "pensar en voz alta" (thinking aloud) se aplica a los estudios de evaluación cualitativa, donde los sujetos, en voz alta, expresan lo que piensan y lo que ocurre en sus mentes durante la ejecución de una tarea. Esas declaraciones son grabadas, observándose, también, el comportamiento de los sujetos en cuanto a expresiones faciales (gestos y movimientos de los ojos). De esta manera, el lenguaje del pensamiento lleva a cabo muchos procesos cognitivos, como la percepción y el raciocinio.

Según Cohen (1984), las técnicas introspectivas son "medidas mentalísticas" que se presentan bajo tres formas básicas: datos provenientes del autorrelato, de la autoobservación y de la autorrevelación.

[...] el autorrelato se refiere a las declaraciones de los individuos sobre cómo creen que realizan ciertas tareas, proporcionadas en situaciones independientes de realización efectiva de la tarea. La autoobservación se refiere a inspecciones de comportamientos específicos durante la realización de una tarea o mientras la información todavía se encuentra sobre el foco de atención [...] o después del evento, retrospectivamente. La autorrevelación no es descripción ni tampoco inspección de comportamientos específicos; es un "pensar en alto" durante la realización de la tarea; el pensamiento es directo y automáticamente exteriorizado; los datos se obtienen sin análisis ni edición.

Scire. $12: 1$ (en.-jun. 2006) 179-195. ISSN 1135-3761. 
En Brasil, entre varios estudios llevados a cabo con el empleo de esa técnica, la tesis doctoral de Nardi (1993) abrió la posibilidad de uso de un instrumento de recogida de datos para la observación de la lectura documental dentro del Grupo de Investigación Análisis Documental de la UNESP-Campus de Marília. La investigación, pionera en este tipo de observación en Brasil y desarrollada por Fujita, Nardi y Fagundes (2003), presenta excelentes resultados en el uso del protocolo verbal, demostrando nuevos aspectos de la lectura documental, entre otras contribuciones.

Con la utilización de la técnica de protocolo verbal, los usuarios "piensan en voz alta", emitiendo sus opiniones y comentarios acerca del objeto evaluado, realizando una evaluación conjunta y participativa: participan en la identificación y entendimiento de los problemas de recuperación de información y utilización del sistema en su propio ambiente de trabajo.

Por considerar de fundamental importancia la observación del usuario para la realización de la evaluación del lenguaje documental DeCS, que posibilita un análisis más efectivo y fiel de sus necesidades informativas, y por la verificación de los importantes resultados obtenidos en investigaciones internacionales llevadas a cabo en el área de las ciencias de la información, se utilizó en este estudio la técnica del protocolo verbal o "pensar en voz alta" (thinking aloud) como instrumento de recogida de datos.

Esa evaluación fue desarrollada de acuerdo con los criterios metodológicos presentados por Ingwersen (1982) y Fujita, Nardi y Fagundes (2003), realizándose algunos ajustes a la realidad de la investigación cuando se consideró necesario. Una de las técnicas aplicadas fue el protocolo verbal individual con escora, esto es, con negociación -interferencia del observador/investigador junto al usuario/investigador (sujeto) durante la aplicación de la técnica.

Según Nardi (1999), el término escora se debe a la existencia de esa interacción entre el investigador y el sujeto en algunos momentos de la realización del protocolo verbal, lo que la propia autora denomina protocolo interactivo o protocolo con escora.

Según Spradley (1980), una observación participativa puede ser entendida dentro de tres universos conceptuales: la participación pasiva, donde el investigador se comporta como simple observador de la situación evaluativa; la participación moderada, donde el investigador tiene la función alterna de observador y participante activo; y la participación activa, donde el investigador desempeña una función de participante en el mayor alto grado de actuación, lo que significa que procura realizar la actividad que los otros participantes están llevando a cabo (realiza la actividad de evaluación y analiza los datos del grupo). 
A este respecto, la participación en el presente estudio fue la de participación moderada, interviniendo en los momentos de recuperación de información, manifestando a los sujetos nuestro grado de satisfacción de los resultados obtenidos, si los términos utilizados en la investigación correspondieron efectivamente a su necesidad informativa y si dichos términos no hubieran podido ser sustituidos por otros disponibles en el lenguaje documental utilizado.

Fue necesario un planeamiento previo de las etapas de trabajo que habrían de ser ejecutadas, así como la adopción de algunos procedimientos que precederían a la aplicación efectiva de la técnica del protocolo verbal para la recogida de datos.

\section{Metodología}

Los procedimientos de aplicación del protocolo verbal se iniciaron con la definición de los sujetos del estudio: los docentes/investigadores del Departamento de Logopedia de la Facultad de Odontología de Bauru de la Universidad de São Paulo, FOB-USP, buscando atender sus necesidades en la realización de investigaciones clínicas básicas y aplicadas al desarrollo y perfeccionamiento de técnicas y métodos diagnósticos y terapéuticos para la mejor atención de la sociedad en general.

La aplicación de esa técnica se llevó a cabo en el propio ambiente de trabajo de los sujetos, tras haber verificado anteriormente el conocimiento de cada uno de ellos del acceso al sistema LILACS, así como la utilización del lenguaje DeCS.

Fueron seleccionados cuatro sujetos, representantes de las cuatro especialidades formadoras del área de la logopedia: lenguaje, audiología, voz y motricidad oral. Se les observó con protocolo verbal en la tarea de realizar la recuperación de información, en el campo descriptor de asunto de la interfaz de búsqueda de la base de datos LILACS, que utiliza el lenguaje DeCS. Los temas investigados fueron definidos por los propios sujetos.

De esa forma, los datos recogidos procedieron de las declaraciones efectuadas por los sujetos durante la realización de la referida actividad, evaluada con la interacción y la intervención moderada del observador/investigador.

Las opiniones emitidas por los sujetos fueron grabadas y posteriormente se realizaron las transcripciones para analizar los datos recogidos.

\section{Resultados y discusión}

Los datos procedentes de las declaraciones de los sujetos participantes en el estudio y los fundamentos teóricos disponibles en la literatura sobre el área de organización de información proporcionaron ayuda para llevar a cabo el análisis de los resultados sobre la realidad temática actual de los términos del vocabulario DeCS correspondientes al campo conceptual de la logopedia.

Scire. $12: 1$ (en.-jun. 2006) 179-195. ISSN 1135-3761. 
Un sistema de información debe proporcionar un acceso inmediato y una rápida recuperación de información que satisfaga las necesidades del usuario y del investigador. Eso es lo que se espera de un sistema que utiliza un lenguaje documental representativo de su área científica.

De acuerdo con Lancaster (2004, p. 19), "un vocabulario controlado es esencialmente una lista de términos autorizados". Por consiguiente, como el mismo autor expone, la función del vocabulario abarca más que eso, es decir, además de ser una lista de términos autorizados, debe controlar los sinónimos y los cuasi sinónimos, diferenciar los términos homógrafos y agrupar los que posean una relación más próxima entre sí. De esta manera se establecen las relaciones jerárquicas, no jerárquicas y de equivalencia.

El análisis de las transcripciones de los protocolos verbales demostró que la metodología empleada fue eficaz por posibilitar la recogida, en tiempo real, de las declaraciones de los sujetos (usuarios/investigadores) sobre el funcionamiento del lenguaje DeCS, utilizado para la recuperación de informaciones en el sistema LILACS.

El estudio condujo a una reflexión sobre las declaraciones emitidas por los cuatro sujetos participantes con la finalidad de proporcionar las condiciones necesarias para seleccionar indicadores de calidad que ayuden a diseñar las estrategias para el perfeccionamiento del vocabulario DeCS en el área de la logopedia.

Los sujetos participantes apuntaron los siguientes motivos para referirse a la ineficacia del lenguaje DeCS, y consecuentemente, a su insatisfacción en la recuperación de información por medio de las búsquedas realizadas en el sistema de información LILACS: insuficiencia de términos genéricos y/o específicos representativos del área de la logopedia; inconsistencia de las relaciones lógico-semánticas entre los términos; clasificación de términos en categorías de asuntos no equivalentes a sus conceptos; necesidad de actualización constante de los términos disponibles en el lenguaje, en relación con la terminología encontrada en la literatura científica del área y adoptada por los especialistas; traducción de diversos términos, notas y definiciones de inglés a portugués, y viceversa, que no corresponden al equivalente utilizado en el área de la logopedia; correspondencia indebida de términos a los significados propuestos; y errores de indización.

\section{Consideraciones finales}

La opción de utilizar un instrumento de recogida de datos introspectivo, centrado en el usuario, permitió realizar de un análisis de los resultados más veraz y fiable en en cuanto a los problemas que presenta el lenguaje documental, debido a que el usuario era el "elemento" fundamental del triángulo sistema de información (con la intermediación del propio lenguaje como instrumento de comunicación)información documental-usuario.

Scire. $12: 1$ (en.-jun. 2006) 179-195. ISSN 1135-3761. 
En este sentido, el presente estudio es innovador y pionero en la utilización de ese instrumento para la evaluación del lenguaje documental en el proceso de recuperación de información en Brasil, donde solo se encontraron referencias a estudios de este tipo llevados a cabo por investigadores en el ámbito internacional del área de las ciencias de la información.

Un lenguaje documental consistente debe reflexionar sobre la terminología empleada en la literatura científica del área, así como sobre la utilizada por el usuario/investigador para la realización de búsquedas satisfactorias y concordantes con la realidad de la investigación.

Según Lancaster (1987), la garantía literaria se fundamenta en el sentido de que un término solo se justifica cuando se tiene realmente conocimiento de la existencia de literatura sobre el asunto; la garantía de uso hace referencia a los términos recogidos a partir de las peticiones de búsqueda realizadas por los usuarios.

La elaboración del lenguaje documental debe también estar de acuerdo con las directrices y normas preestablecidas y disponibles en el área en lo que respecta a la forma de presentación de sus términos y de su estructura lógico-semántica, entre otros segmentos necesarios para su consistencia en el momento de la recuperación de información.

Los casos indicados por los sujetos participantes y presentados en este estudio no correspondieron a los conceptos presentados por la literatura, proporcionando de esta manera la base necesaria para la identificación de los principales elementos causantes de la ineficacia del lenguaje.

En este sentido y de acuerdo con la situación real en que se encontraron los términos de logopedia constituyentes del lenguaje documental DeCS, se recomienda al BIREME (Centro Latinoamericano y del Caribe de Información sobre Ciencias de la Salud) la construcción de una categoría específica para el área de la logopedia, dado que ese campo temático tiene características propias dentro del área de ciencias de la salud de Brasil, posibilitando la correspondencia entre la representación terminológica y las necesidades de los usuarios/investigadores brasileros para la recuperación de información que contribuya a la creación de conocimientos y a la construcción de la ciencia brasilera.

La medida se considera también totalmente viable por el hecho de que el lenguaje DeCS ya presenta las adaptaciones necesarias al incluir las categorías de salud pública y homeopatía, inexistentes en el lenguaje documental de origen, el MeSH.

Dentro del contexto de trabajo en coordinación llevado a cabo por el BIREME en la prestación de sus servicios por medio de la comunidad especializada en la administración, tratamiento y difusión de la información - los centros colaboradores-, se debe elaborar una categoría específica para el área de la logopedia, dentro de una filosofía de trabajo de participación e integración. 
De esta manera, los especialistas/investigadores del área, los profesionales de la información que posean experiencia en la actividad de indización y/o recuperación de información dentro de esta temática, así como conocimientos sobre la elaboración de lenguajes documentales, y las entidades de investigación como la Sociedad Brasilera de Logopedia son recursos humanos e institucionales que reúnen todas las condiciones para el desarrollo de esa categoría disciplinar.

Para tales fines, se establecieron algunos indicadores de calidad como criterios para la elaboración de la categoría de logopedia dentro de la estructura/relación multijerárquica del vocabulario:

- Recopilación y estandarización de una terminología del área de la logopedia utilizada por especialistas (usuarios/investigadores), entidades de investigación y literatura científica que represente de la manera más fiel y amplia posible las especialidades de lenguaje, audiología, voz y motricidad oral dentro de los principios de generalización y especificidad de los términos y de las directrices y normas existentes.

- Actualización de los descriptores ya existentes en cuanto a terminología y definiciones, para aproximarse a la realidad científica de la logopedia brasilera.

- Revisión de las relaciones lógico-semánticas entre los términos existentes y establecimiento de este tipo de relaciones entre los nuevos términos que se integren al vocabulario.

- Control más efectivo de términos sinónimos y casi sinónimos, para evitar la dispersión temática y proporcionar una mayor exactitud en la indización de contenido y una recuperación más eficaz de la información.

- Expansión de las áreas de educación, psicología, lingüística, neurolingüística, medicina (otorrinolaringología), odontología, física e salud pública para atender las necesidades de correspondencia temática de esos campos conceptuales con la logopedia, teniendo en cuenta la naturaleza multidisciplinar del área.

- Recopilación de las necesidades de incorporación de otros términos de áreas afines a la logopedia, como anatomía, fisiología o bioquímica, entre otras.

- Revisión de la traducción de todos los términos existentes en el vocabulario DeCS — de inglés y español a portugués— de modo que exista correspondencia entre los conceptos y los términos empleados por la literatura de la logopedia brasilera.

- Realización de la traducción de portugués a inglés y español de todos los nuevos términos, de acuerdo con el uso terminológico empleado por las comunidades científicas norteamericana y latinoamericana.

Scire. $12: 1$ (en.-jun. 2006) 179-195. ISSN 1135-3761. 
- Revisión de las “notas de definición”, verificación de las acepciones de cada término y aplicación de dichas notas/definiciones a todos los términos que integren la categoría.

- Revisión de las "notas de indización" y aplicación de dichas notas, cuando sea necesario, a los nuevos términos.

- Aplicación de los calificadores cuya utilización se considere "permitida" para los nuevos términos.

Con la adopción de esos indicadores de calidad, se tenderá a la elaboración de un lenguaje documental estructurado y representativo de la cultura de su sistema de información, que proporcionará una consistencia mayor de las informaciones almacenadas y recuperadas, así como un aumento de la eficacia del índice temático y, consecuentemente, de la recuperación de información (Santos, 2002).

Esa medida posibilitará, también, un mejor funcionamiento de la terminología latinoamericana junto al proyecto de desarrollo de una terminología única para la salud —UMLS, Unified Medical Language System: http://www.nlm.nih.gov/research/umls/—, propuesto por la United States National Library of Medicine, teniendo el BIREME la responsabilidad de la actualización y el paso de los términos a portugués y español.

\section{Notas}

(1) Descriptores en Ciencias de la Salud.

(2) Literatura Latinoamericana y del Caribe sobre Ciencias de la salud.

(3) Centro Latinoamericano y del Caribe de Información en Ciencias de la Salud.

\section{Referencias}

Austin, Derek; Dale, Peter (1993). Diretrizes para o estabelecimento e desenvolvimento de tesauros monolingues. Tradução de Bianca Amaro de Melo. Brasília: IBICT, 1993.

BIREME, Centro Latinoamericano e do Caribe de Informação em Ciências da Saúde (2004a). Fundamentos, missão, objetivos, estrutura organizacional. URL: <http://www.bireme.br/ bvs/bireme/P/objetivos.htm>. Consultado: 2004-12-29.

BIREME, Centro Latinoamericano e do Caribe de Informação em Ciências da Saúde (2004b). O que são vocabulários estruturados? URL: <http://decs.bvs.br/P/about vocabp.htm>. Consultado: 2004-09-30.

Brasil. Ministério da Saúde. Conselho Nacional de Saúde (2004). Manual da $2^{\mathrm{a}}$ Conferência Nacional de Ciência, Tecnologia e Inovação em Saúde. Brasília DF, Jul. 25-28, 2004. Brasília, DF: Conselho Nacional de Saúde, Ministério da Saúde, 2004.

Cleverdon, Cyril W. (1964). Evaluation of operational information retrieval systems. Part 1: Identification of criteria. Cranfield: College of Aeronautics, 1964.

Scire. $12: 1$ (en.-jun. 2006) 179-195. ISSN 1135-3761. 
Cohen, Andrew D. (1987). Using verbal reports on research on language learning. // Faerch, Claus; Kasper, Gabriele (eds.) (1987). Introspection in second language research. Clevedon: Multilingual Matters, 1987 (apud Nardi, 1999, p. 123).

Conselho Federal de Fonoaudiologia (2001). Resolução CFF nº 269, de 3 de março de 2001: dispõe sobre as especialidades reconhecidas pelo Conselho Federal de Fonoaudiologia, e dá outras proviodências. URL: <http://www.Fonoaudiologia.org.br/resolu/ RESOL269.HTM>. Consultado: 2005-02-02.

Ericsson, K. Anders; Simon, Herbert A. (1987). Verbal reports on thinking. // Faerch, Claus; Kasper, Gabriele (eds.). Introspection in second language research. Clevedon: Multilingual Matters, 1987. 24-53.

Foskett, Antony Charles (1973). A abordagem temática da informação. Tradução de Antonio Agenor Briquet de Lemos. São Paulo: Polígono, 1973.

Fujita, Mariângela Spotti Lopes; Nardi, Maria Isabel Asperti; Fagundes, Silvana Aparecida (2003). A observação da leitura documentária por meio de protocolo verbal. // Rodrigues, Georgette Medleg; Lopes, Ilza Leite (coords.). Organização e representação do conhecimento na perspectiva da ciência da informação. Brasília: Thesaurus, 2003. 141-178. (Estudos avançados em ciência da informação, v. 2).

Ingwersen, Peter (1982). Search procedures in the library: analysed from the cognitive point of review. Journal of Documentation. 38:3 (September 1982) 165-191.

Jiménez Miranda, Jorgelina (1998). Acceso a MEDLINE y LILACS mediante el MeSH e el DeCS. ACIMED. 6:3 (septiembre-diciembre 1998) 153-162. URL: <http://scielo. sld.cu>. Consultado: 2005-06-21.

Jiménez Miranda, Jorgelina (2002). La indización en el Sistema Nacional de Información de Ciencias Médicas. Parte II: Descriptores en Ciencias de la Salud (DeCS). ACIMED. 10:4 (julio-agosto 2002). URL: <http://scielo.sld.cu>. Consultado: 2005- 06-21.

Lancaster, Frederick Wilfrid (1987). Construção e uso de tesauros: curso condensado. Trad. de César Almeida de Meneses Silva. Brasília: IBICT, 1987.

Lancaster, Frederick Wilfrid (2004). Indexação e resumos: teoria e prática. Trad. de Antonio Agenor Briquet de Lemos. Brasília: Briquet de Lemos, 2004.

Melo, Luiza Baptista (1994). Avaliação de sistemas de recuperação de informação: breve retrospectiva dos principais projetos. Cadernos BAD. 2 (1994) 29-46.

Nardi, Maria Isabel Asperti (1993). As expressões metafóricas na compreensão de texto escrito em língua estrangeira. Conferencia. São Paulo: Pontifícia Universidade Católica, 1993.

Nardi, Maria Isabel Asperti (1999). A metáfora e a prática de leitura como evento social: instrumentos do pensar a biblioteconomia do futuro. Tesis. São Paulo: Pontifícia Universidade Católica de São Paulo, 1999.

Otero, P., et al (2004). Evolution of medical informatics in bibliographic databases. Medinfo. 11:1 (2004) 301-305.

Pellizzon, Rosely de Fátima (2004). Pesquisa na área de saúde: 1. Base de dados DeCS (Descritores em Ciências da Saúde). Acta Cirúrgica Brasileira. 19:1 (marzo-abril 2004) 153163. URL em: <http://www.scielo.br>. Consultado: 2004-09-29. 
Rodríguez Camiño, Reinaldo (1998). MeSH o DeCS: algunas consideraciones sobre la indización biomédica. ACIMED. 6:3 (septiembre-diciembre 1998) 163-170. URL: $<$ http://scielo.sld.cu>. Consultado: 2005-06-21.

Santos, Cibele Araújo Camargo Marques dos (2002). Linguagens documentárias e codificação da informação: estudo de vocabulário da área de saúde. Conferencia. São Paulo: Escola de Comunicação e Artes, Universidade de São Paulo, 2002.

Spradley, James P. (1980). Participant observation. Nueva York: Holt, Rinehart \& Winston, 1980.

Strehl, Letícia (1998). Avaliação da consistência da indexação realizada em uma biblioteca universitária de artes. Ciência da Informação. 27:3 (septiembre-diciembre 1998) 329-355.

United States National Library of Medicine (2005). Medical Subject Headings - MeSH. URL: <http://www.nlm.nih.gov/mesh/MBrowser.html>. Consultado: 2005-03-13.

Valdés Abreu, Manuela de la C. (1996). Necesidad de las notas de alcance de los calificadores en la "Introducción" del tesauro Descriptores en Ciencias de la Salud (DeCS). ACIMED. 4:1 (enero-abril 1996) 23-28. URL: <http://scielo.sld.cu>. Consultado: 2005-06-21.

Scire. $12: 1$ (en.-jun. 2006) 179-195. ISSN 1135-3761. 